\title{
Percepção dos pais sobre as práticas de educação em saúde na Unidade Neonatal
}

\author{
Parents' perception of health education practices in Neonatal Unit \\ Percepción de los padres sobre las prácticas de educación para la salud en una Unidad Neonatal
}

Nisa Rubina Pereira Souto Rosa ${ }^{1,2}$ (1)

Maria Alice dos Santos Curado ${ }^{1}$ (1)

Maria Adriana Pereira Henriques ${ }^{1}$ [C]

1. Escola Superior de Enfermagem de Lisboa, Centro de Investigação, Inovação e Desenvolvimento em Enfermagem de Lisboa. Lisboa, Portugal.

2. Hospital Dr. Nélio Mendonça. Funchal, Portugal.
Autor correspondente:

Nisa Rubina Pereira Souto Rosa

E-mail: nisa.souto@hotmail.com

Recebido em 24/03/2021.

Aprovado em 29/07/2021.

DOI:https://doi.org/10.1590/2177-9465-EAN-2021-0040

\section{Resumo}

Objetivo: analisar a percepção dos pais sobre as práticas de educação em saúde desenvolvidas pelos enfermeiros na Unidade Neonatal que facilitaram a aquisição de competências parentais para uma tomada de decisão fundamentada. Método: estudo exploratório e descritivo, com abordagem qualitativa. Participaram 13 pais com filhos internados, pela primeira vez, em uma Unidade Neonatal portuguesa. Os dados foram colhidos entre fevereiro e agosto de 2020, por meio de grupos focais, processados no software Interface de R pour Analyses Multidimensionnelles de Textes et de Questionneires, através da Classificação Hierárquica Descendente e da Análise de Similitude. Resultados: da Classificação Hierárquica Descendente, emergiram quatro classes: "Necessidades de informação", "Lacunas na informação", "Disponibilidade para informar"; "Práticas facilitadoras da educação em saúde". Conclusão e implicações para a prática: os pais consideraram como práticas facilitadoras da educação em saúde a tecnologia digital aliada à disponibilidade dos enfermeiros para informar e explicar, bem como propiciar um ambiente empático e informal. Tal resultado pode subsidiar o desenvolvimento de intervenções de enfermagem de educação em saúde para pais na Unidade Neonatal, com recurso à tecnologia digital.

Palavras-chave: Neonatologia; Enfermagem; Pais; Educação em Saúde; Letramento em Saúde.

\section{Abstract}

Objective: to analyze parents' perception of health education practices developed by nurses in a Neonatal Unit that facilitated the acquisition of parenting skills for an informed decision-making. Method: this is an exploratory, descriptive and qualitative study. We included 13 parents with children hospitalized for the first time in a Portuguese Neonatal Unit. Data were collected between February and August 2020, through focus groups, processed in the software Interface de R pour Multidimensionnelles de Textes et de Questionneires, through Descending Hierarchical Classification and Similitude Analysis. Results: four classes emerged from the Descending Hierarchical Classification: "Information needs"; "Information gaps"; "Availability to inform"; "Facilitating health education practices". Conclusion and implications for practice: parents considered digital technology combined with nurses' availability to inform and explain, as well as provide an empathetic and informal environment as facilitating practices for health education. These results can support health education nursing interventions for parents at the Neonatal Units using digital technology.

Keywords: Neonatology; Nursing; Parents; Health Education; Health Literacy

\section{Resumen}

Objetivo: analizar la percepción de los padres sobre las prácticas de educación en salud desarrolladas por enfermeras de la Unidad Neonatal que facilitaron la adquisición de habilidades parentales para la toma de decisiones informada. Método: estudio exploratorio descriptivo, con abordaje cualitativo. Participaron 13 padres con hijos hospitalizados, por primera vez, en una Unidad Neonatal Portuguesa. Los datos fueron recolectados entre febrero y agosto de 2020, a través de grupos focales, procesados en el software Interface de $R$ pour Multidimensionnelles de Textes et de Questionneires, a través de la Clasificación Jerárquica Descendente y el Análisis de Similitud. Resultados: surgieron cuatro clases de la Clasificación Jerárquica Descendente: "Necesidades de información”; "Brechas de información”; "Disponibilidad para informar”; "Prácticas facilitadoras de la educación para la salud”. Conclusión e implicaciones para la práctica: los padres consideraron prácticas facilitadoras para la educación en salud la tecnología digital combinada con la disponibilidad de enfermeras para informar y explicar, además de brindar un ambiente empático e informal. Este resultado puede apoyar el desarrollo de intervenciones de enfermería de educación para la salud para los padres en Unidad Neonatal, con el uso de tecnología digital.

Palabras clave: Neonatología; Enfermería; Padres; Educación para la Salud; Alfabetización en Salud. 


\section{INTRODUÇÃO}

O internamento em uma Unidade Neonatal (UN) pode afetar a identidade e o desempenho de tarefas parentais ${ }^{1}$. Assim, as abordagens do Cuidado Centrado na Família (CCF) são as privilegiadas neste contexto, pois promovem um cuidado colaborativo e em parceria com os pais e uma tomada de decisão informada².

A essas abordagens, está subjacente um conhecimento das preferências e das necessidades dos pais. Entre as necessidades parentais identificadas, as relativas à informação têm merecido destaque em estudos científicos ${ }^{3,4}$. Os pais expressam a necessidade de obter informação precisa, atempada e compreensível, para se sentirem competentes para cuidar do filho em casa ${ }^{5}$. Nesse âmbito, diversos programas têm sido implementados, contudo a sua adoção permanece um desafio ${ }^{6}$. A investigação científica tem contribuído para identificar fatores que facilitem ou inibam essas práticas. Um fator facilitador é a confiança dos pais na fonte de informação ${ }^{7}$, sendo os profissionais de saúde identificados como a fonte mais fidedigna ${ }^{8}$ e a equipe de enfermagem como a melhor fonte de informação, por dedicar mais tempo a explicar e a validar a compreensão ${ }^{6}$. A capacidade de os pais compreender e assimilar a informação é outro fator facilitador ${ }^{7}$. Essa capacidade é afetada pelo stresse associado ao internamento, pela quantidade de informação especializada que é transmitida ${ }^{9}$ e pelo nível de Letramento em Saúde (LS) parental ${ }^{10}$. A informação pode, inclusive, tornar-se inútil quando não é transmitida atempadamente, não é monitorizada a compreensão ou quando os esclarecimentos são inconsistentes ${ }^{6}$. Perante o exposto, torna-se imprescindível uma prática de educação em saúde que colmate esses fatores inibidores.

A educação em saúde organizada e estruturada tem sido considerada a principal via para melhorar a LS, ou seja, a capacidade da pessoa aceder, compreender, avaliar e usar a informação em saúde para tomar decisões, de acordo com as suas escolhas, e implementar ações acertadas em saúde ${ }^{11}$. Porém, requer uma abordagem que englobe todos os níveis de LS (funcional, interativa e crítica) e não apenas o funcional, com o recurso a metodologias mais interativas, que não se limitem à transmissão diretiva da informação, mas que sejam empoderadoras ${ }^{12}$. Para além das capacidades individuais e das condições sociais, a capacidade de interpretar informação em saúde irá depender da facilidade com que esta é apresentada pelos sistemas de saúde ${ }^{13}$. Tradicionalmente, a prática de educação em saúde é caracterizada pela transmissão oral de informação, pela demonstração e prática com os profissionais de saúde, complementada com informação impressa ${ }^{6}$. Atualmente, observamos outras práticas associadas à expansão das tecnologias digitais, como módulos educativos online e aplicações móveis (app), com o potencial de reduzir as lacunas de acesso ao conhecimento ${ }^{14}$. Uma revisão scoping sobre a efetividade das intervenções de comunicação entre pais e profissionais nas UN revelou o interesse em implementar essas tecnologias para promover o $\mathrm{CCF}^{15}$ e a preferência e receptividade dos pais à sua utilização ${ }^{16}$.
Perante a multiplicidade de potenciais fatores inibidores à educação em saúde dos pais na UN, há a necessidade de uma maior compreensão sobre como os enfermeiros poderão tornar essas práticas mais adequadas às necessidades de educação dos pais, quais as que os pais consideram ser facilitadoras e em que medida as tecnologias digitais podem ser uma mais-valia. Desse modo, o presente estudo tem como objetivo analisar a percepção dos pais sobre as práticas de educação em saúde desenvolvidas pelos enfermeiros na UN que facilitaram a aquisição de competências parentais para uma tomada de decisão fundamentada.

Os seus resultados permitirão preparar um estudo subsequente sobre uma intervenção de educação em saúde que contribua para promover a LS dos pais na UN, com recurso a app.

\section{MÉTODO}

\section{Tipo de estudo}

Trata-se de um estudo exploratório e descritivo, com abordagem qualitativa, com dois grupos focais (GF) realizados em fevereiro e agosto de 2020. Na entrevista de grupo, os dados são gerados pelo debate em grupo sobre o fenômeno em estudo dinamizado pelo moderador ${ }^{17}$ e permite o aprofundamento e o contraste de diferentes perspetivas, opiniões e experiências entre pessoas semelhantes ${ }^{18}$.

\section{Amostra}

Participaram no estudo 13 pais que tiveram filhos internados, pela primeira vez, em uma UN.

\section{Critérios de seleção}

Foram estabelecidos como critérios de inclusão: serem pais (mãe ou pai) de nacionalidade portuguesa, que dominem a língua portuguesa e que tiveram, nos últimos seis meses, um filho internado em UN. Aqueles pais que não atenderam o telefone após três contatos ou não tiveram disponibilidade na data agendada foram excluídos. Por considerar que a duração do internamento poderá influenciar na percepção dos pais sobre as práticas de educação em saúde, no primeiro GF, o internamento na UN foi superior a 15 dias, e no segundo, foi inferior a 15 dias. Para a seleção dos pais, utilizaram-se as variáveis sexo, duração do internamento na UN e a primeira experiência de internamento na UN. Essa diversificação dos participantes, integrados no GF, permitiu cumprir o pressuposto da heterogenidade dos pais. O recrutamento dos participantes foi efetuado telefonicamente e complementado com um convite via correio eletrõnico.

\section{Coleta de dados}

Os GF ocorreram em uma sala privada e contaram com um moderador (investigador principal) e um assistente com funções de observação da comunicação não verbal, gestão do tempo e registo de informações pertinentes ${ }^{17}$. Os métodos de coleta de dados incluíram a gravação audiovisual, complementada com as anotações e a observação participativa realizadas pelo assistente. 
Os instrumentos de coleta de dados foram guia semiestruturado, questionário de caracterização dos participantes e grade de observação. No início de cada GF, o moderador apresentou os objetivos pretendidos e as regras gerais do GF. No final, foi realizada uma breve síntese sobre os temas debatidos, o que permitiu resumir e clarificar as principais conclusões. Os dois GF tiveram a duração média de 60 minutos.

\section{Análise e tratamento dos dados}

Os dados demográficos foram submetidos à análise descritiva. O verbantim dos registros de áudio foram transcritos e, posteriormente, analisados e complementados com as informações advindas da gravação audiovisual e das anotações efetuadas. Para tratar os dados, utilizou-se o software Interface de $R$ pour Analyses Multidimensionnelles de Textes et de Questionneires (IRAMUTEQ $®$ ), versão 0.7 Alpha $2^{19,20}$, que realizou uma análise lexicográfica utilizando a Classificação Hierárquica Descendente (CHD) e Análise de Similitude (AS). A análise dos dados foi feita pelo pesquisador e ocorreu a partir do referencial teórico do $\mathrm{CCF}^{2}$.

\section{Aspetos éticos}

O estudo foi aprovado pelo Comitê de Ética em Pesquisa da Instituição Hospitalar, em 03/07/2019, sob parecer E.E909519. Todos os participantes foram informados oralmente e por escrito que a sua participação era voluntária, que poderiam deixar de participar a qualquer momento sem quaisquer penalização $e$ que a confidencialidade e anonimato dos dados seriam sempre garantidos. O Consentimento Informado, Livre e Esclarecido foi assinado por todos, previamente à sua participação.

\section{RESULTADOS}

Dos 18 pais elegíveis, cinco foram excluídos por não comparecência, pelo que participaram nos GF sete mães e seis pais, com uma média de idades de 29 anos. Quanto ao nível de escolaridade, $38,5 \%$ têm ensino superior, $23,1 \%$ têm ensino secundário e $38,5 \%$ têm ensino básico. Eram pais pela primeira vez $53,8 \%$ dos participantes, enquanto $46,1 \%$ já tinham um filho. A idade gestacional à nascença do filho variou entre 26 e 35 semanas.

A análise lexicográfica do corpus textual proveniente da transcrição dos dois GF foi composto por 30 textos organizados, de forma monotemática, em um arquivo único no programa Apache OpenOffice. Cada um deles foi separado por uma linha de comando e caracterizado pelas variáveis sexo $(p)$, número de filhos (f) e nível de escolaridade (e) (Ex: ${ }^{* * * \star}$ ${ }^{*} \mathrm{q} \_1{ }^{*} \mathrm{fg} \_1{ }^{*} \mathrm{~s} \_2{ }^{*} \_2{ }^{*} \mathrm{e} \_2$ ), para as três questões (q) do GF: Q1 - necessidade de informação, Q2 - práticas de educação em saúde, Q3 - utilização de tecnologias digitais. Obtiveram-se 112 segmentos de texto, dos quais $99(88,39 \%)$ foram aproveitados pelo IRAMUTEQ $®$. Este resultado indica uma boa consistência e adequação do conteúdo submetido para este tipo de análise ${ }^{20,21}$ Foram contabilizadas 889 palavras, que ocorreram 3.761 vezes, com uma frequência média de 33,58 palavras para cada forma.
O ponto de corte utilizado para a inclusão das palavras nas classes do dendrograma foi 0,67 .

A CHD identificou quatro classes que foram nomeadas a partir dos seus descritores. A primeira repartição de conteúdo demarca as Classes 3 (Necessidades de informação), 2 (Lacunas na informação) e 1 (Disponibilidade para informar) da Classe 4 (Práticas facilitadoras da educação em saúde). A segunda repartição delimita a Classe 3 das Classes 2 e 1. Para melhor compreender o processo de divisão do conteúdo textual e da constituição das classes, apresentamos a Figura 1 (dendrograma da $\mathrm{CHD}$ ), com os vocábulos associados a cada classe que atenderam ao critério de qui-quadrado $\geq 3,84$ e $p$-value inferior a $0,05^{20}$

Classe 3 compreende $26,26 \%$ do corpus total analisado. A percepção dos pais sobre a informação na UN está relacionada com os vocábulos panfleto, falar, horário, entregar, choque, preparar, procurar, participar, apanhar, entrar e conta-gotas. Os discursos dos pais nessa classe remeteram para a necessidade, a adequabilidade e o modo como obtiveram a informação. Em sua maioria, os pais expressaram satisfação com a informação transmitida na UN: eu fui sempre informado (fg2), ter informação facilitou (fg1) e as informações foram transmitida nas alturas corretas (fg1). Um dos pais manifestou que a informação foi dada a conta-gotas (fg2), o que o fazia pensar que a filha não estava bem: estava a regredir. Os pais também referiram a necessidade de melhorar a preparação antes de entrar na UN ou do acolhimento na primeira visita: poderiam explicar à mãe e ao pai o que poderia vir a acontecer para nós nos prepararmos psicologicamente (...) ter mais informação, se calhar, já não ía sofrer tanto ( $\mathrm{fg} 1$ ). Os pais relataram que o primeiro contato com a UN foi um choque, o que dificultou a comunicação com os profissionais de saúde e, sobretudo, a compreensão da informação: [os profissionais] falaram, falaram, mas não entrou nada (fg1), falaram não sei o quê, não me lembro, essa parte não me lembro (fg1). Na sua maioria, os pais mencionaram que o choque foi atenuado pela experiência prévia de internamento de um familiar em uma unidade de cuidados intensivos e pela possibilidade de ver a foto do filho antes de ver presencialmente, pois ajuda a estar mais preparado e não apanhar logo aquele primeiro choque (fg2).

Reforçam ainda que a entrega de panfletos como complemento da informação transmitida lhes permitiu rever alguma da informação fornecida. Quanto ao panfleto de acolhimento, os pais enfatizaram os horários (fg2) das visitas e a informação sobre a unidade (fg1). Duas mães fizeram referência ao panfleto alusivo ao Método Canguru (fg1) e outra mãe referiu que o único panfleto que me foi entregue foi o teste do pezinho (fg2). Uma mãe mencionou que ainda tinha em casa o panfleto sobre o aleitamento materno e salientou a sua utilidade para saber a parte da congelação (fg1). Contudo, outros pais referiram que não entregaram nenhum panfleto (fg2) durante o internamento na UN, o que pode estar ligado ao choque inicial do primeiro contato com a UN. Os pais também procuraram informação, especificamente na Internet: eu pesquisei por mim (fg2); cheguei a ir à Internet 


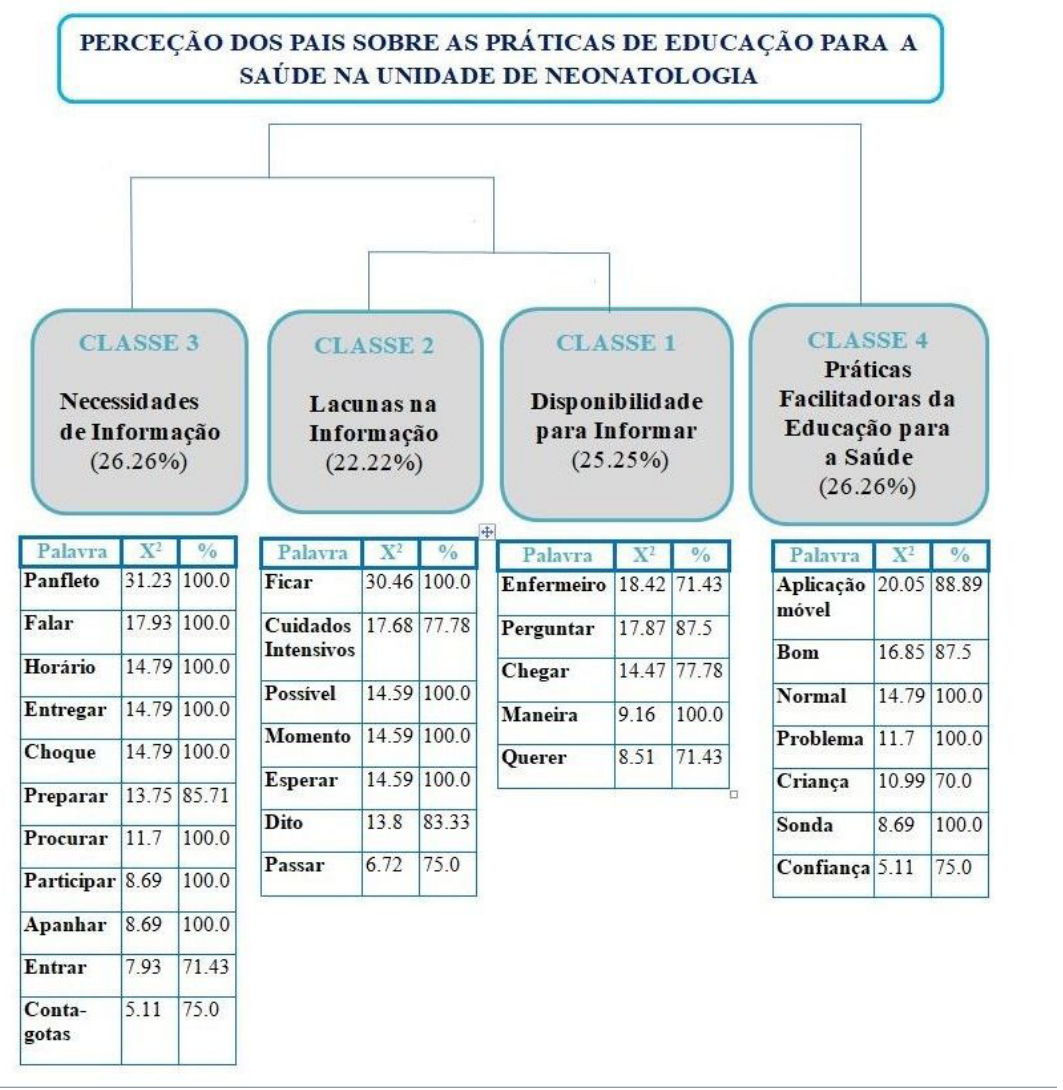

Figura 1. Classes componentes do dendrograma do corpus textual. Elaborado pelos autores. Portugal, 2020

e procurei muita coisa (fg1), procuramos informação no doutor Google (fg1). Esta pesquisa, por um lado, foi considerada útil: procurarna Internet desmistificou algumas coisas (fg1); mas, por outro lado, também gerou alguma confusão: é interessante mas também há muita confusão (fg1). Uma mãe referiu que recorreu a uma familiar enfermeira como fonte de informação, enquanto um pai mencionou que não foi procurar informação a nenhum sítio, mas acredito que seja necessário (fg1).

A Classe 2 constitui $22,22 \%$ do corpus total analisado. Os vocábulos associados foram ficar, cuidados intensivos, possivel, momento, esperar, dito e passar. Nessa classe, os discursos dos pais remeteram a aspetos que poderiam ser melhorados. Consideraram que seria importante visitar antes a unidade principalmente nas situações que é possível antever (fg1), de modo a conhecer a unidade, a equipe e saber que estavam preparados para receber a filha. Mencionaram falta de informação sobre a duração do internamento, pois, ao mesmo tempo que estão a dizer que está tudo bem, estão a prolongar o internamento (fg2) sobre a admissão na UN, pois não nos foi dito que ela ia para os cuidados intensivos (fg2), disseram que ia fazer exames, o pai pelas letras apercebeu-se que eram os cuidados intensivos (fg2) e sobre a possilidade de amamentar na UN (fg2).

A Classe 1 compreende $25,25 \%$ do corpus total analisado e foi denominada "Disponibilidade para informar", com base nos vocábulos enfermeiro, perguntar, chegar, maneira e querer. Nessa classe, constatou-se que os pais valorizaram a informação dada pelos enfermeiros e o fato de se disponibilizarem para explicar e esclarecer logo as dúvidas, independentemente da hora. Os pais mencionaram que, mesmo sem perguntarlá, vinha o enfermeiro ao meu encontro e dizia ela está assim porque aconteceu isto, e isto (fg2) e que explicavam na hora, se não fosse na hora quando tivesse um médico ou enfermeiro que soubesse da situação explicava (...) acho que só o facto de nos perguntarem também nos fazia querer fazer mais e melhor (...) e são estas aprendizagens que depois levamos para casa (fg1). Outro pai também valorizou a disponibilidade dos enfermeiros mesmo durante a noite (fg2).

Por fim, a Classe 4 foi denominada "Práticas facilitadoras da educação em saúde" e corresponde a $26,26 \%$ do corpus total analisado. Essa classe reuniu os vocábulos aplicação móvel, bom, normal, problema, criança, sonda e confiança. Os discursos dos pais revelam que esses valorizam outras formas de transmitir a informação, como ter acesso a app da UN: faria todo o sentido (fg1), ía ajudar (fg2), era uma boa ideia (fg2), em especial se fosse uma app da unidade temos outra confiança (fg1), temos a segurança da informação ser fidedigna (fg1), o que não acontece com o que encontramos na Internet. Um dos pais salientou que ter uma app seria útil nos internamentos por poucos ou muitos dias (fg2) e que poderia conter informação 


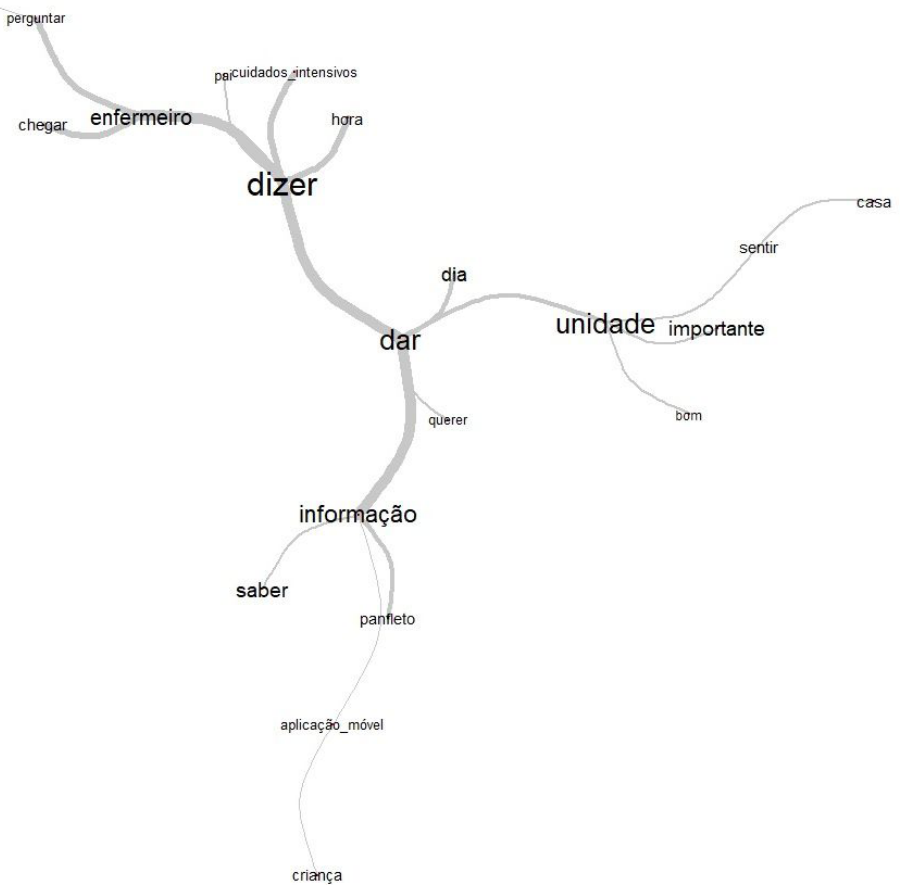

Figura 2. Análise de Similitude do corpus textual. Elaborado pelos autores. Portugal, 2020

sobre a nossa criança (...) os vários tipos de problemas que poderiam acontecer durante a estadia (fg1), uma secção pessoal com os dados da própria criança (...) informações genéricas do que é uma intercorrência normal de um prematuro (fg1). Outras práticas consideradas facilitadoras foram o envio de mensagens de texto via telemóvel, com o estado da criança (...) escrever bom dia mãe hoje estou assim (...), o ter acesso a imagens da criança em tempo real (fg2) e um vídeo para saber que existe uma carga de fios ali ligados à criança e sondas e tudo o que é completamente normal (fg1). Os pais também valorizaram a participação ativa nos cuidados, foi muito bom e isso é depois o que supera a expectativa (fg1), bem como o ambiente informal, pois sentir que a criança está em casa, ambiente menos formal dá confiança ( $\mathrm{fg} 1)$.

A AS sintetiza o conteúdo lexical apreendido a partir das classes apresentadas. Nesta análise, a maior nitidez das ligações entre os vocábulos traduz a sua relevância para a compreensão do fenômeno estudado. Essa foi pautada pela coocorrência entre os vocábulos dizer, dar, informação e unidade, ilustrado na Figura 2.

O vocábulo dizer foi o mais recorrente e apresenta maior correlação com dar e enfermeiro e menor com hora e cuidados intensivos. Do vocábulo dar, ramificam-se informação e unidade. Assim, os resultados demonstram que, associado à informação, estão os vocábulos saber, panfleto e aplicação móvel, que revelam as práticas que os pais consideram ser facilitadoras; relacionados com unidade, encontram-se os vocábulos bom, importante, sentir e casa, que expressam o que os pais valorizam.
Ao vocábulo enfermeiro, estão associados chegar, perguntar e explicar, que correspondem à disponibilidade demonstrada por este profissional.

\section{DISCUSSÃO}

A análise da percepção dos pais sobre as práticas de educação em saúde realizadas pelos enfermeiros na UN permitiu identificar necessidades e lacunas na informação transmitida, práticas que poderão facilitar a aquisição de competências parentais para uma tomada de decisão informada, bem como a atitude de disponibilidade dos enfermeiros para os informar.

$\mathrm{Na}$ Classe 3, os pais valorizaram a informação e salientaram a importância de ser ponderada a quantidade e o momento em que esta é transmitida, pois influencia a sua preparação e controle emocional. Esses resultados são corroborados por uma revisão de literatura ${ }^{21}$, que evidenciou que as necessidades de informação destes pais são elevadas, dinâmicas e que, quando incompletas ou de difícil compreensão, aumentam o seu stresse. Os pais do presente estudo manifestaram a necessidade de uma maior preparação para entrar pela primeira vez na UN, decorrente do choque emocional que sentiram e que os impediu de recordar a informação e enfatizaram como benéfica a disponibilização prévia de uma foto do filho, o que é condizente com os resultados do estudo de revisão de Brett et al. ${ }^{6}$. Os pais também valorizaram os panfletos informativos entregues pelos enfermeiros, que também foi considerada uma intervenção efetiva para comunicar, apoiar e providenciar informação aos pais de recém-nascidos 
prematuros no mesmo estudo de revisão 6 . Contudo, manifestaram a necessidade de informação complementar e, para obtê-la, recorreram a familiares da área da saúde e à Internet. Esse fato é condizente com os resultados do estudo de Lima e Mazza $(2019)^{4}$, que identificou que as necessidades informativas dos pais são amplas e que estes recorrem a recursos tradicionais, mas também tecnológicos, para satisfazê-las.

As Lacunas na informação (Classe 2) identificadas pelos pais foram a inexistência de uma visita prévia à UN para conhecer a equipe e o local e a falta de informação sobre a admissão e duração do internamento. Essas lacunas encontram relação com os resultados do estudo de revisão de Brett et al. ${ }^{6}$, que fizeram referência às potencialidades da visita prévia à UN, e do estudo de Lebel et al. ${ }^{14}$, que evidenciou ser benéfico para os pais receber informação antes do nascimento e admissão na UN e no decurso do internamento. A falta de informação sobre a admissão e duração do internamento foram igualmente reportados na revisão scoping de Ballantyne et al. ${ }^{3}$ como uma fonte de stresse associada à ausência de envolvimento na decisão e de informação adequada para preparar para a transição.

Por sua vez, a disponibilidade dos enfermeiros para informar e explicar (Classe 1) foi também valorizada no estudo de Williams et al. ${ }^{22}$, que relacionou a redução do stresse parental com a quantidade e qualidade da comunicação entre os profissionais de saúde e os pais. Ao investigar as necessidades de informação das famílias na UN, Lima e Mazza ${ }^{4}$ concluiram que os pais desejam que os profissionais dediquem tempo a explicar, esclarecer dúvidas sobre termos técnicos em uma base regular. Por sua vez, o estudo de revisão de Brett et al. ${ }^{6}$ revelou que os pais privilegiavam os enfermeiros como fonte de informação, por dedicarem mais tempo a explicar e a validar a compreensão. Essa atitude de disponibilidade dá suporte ao preconizado nas abordagens de $\mathrm{CCF}^{2}$.

Por fim, os pais identificaram como Práticas facilitadoras de educação para a saúde (Classe 4) o uso da tecnologia digital, nomeadamente através de app da UN, envio de mensagem de texto, de vídeo em tempo real ou de filmagens do filho e da UN. Esses resultados estão alinhados com outros estudos, que revelaram que os pais desejam e consideram benéfico disponibilizar uma app neonatal na $\mathrm{UN}^{14}$ e que preferiam os recursos digitais em detrimento dos impressos, em especial, os indicados pelos profissionais da UN, por serem mais fidedignos ${ }^{16}$. O envio de mensagens de texto com informação sobre o filho foi igualmente referido como facilitador em outro estudo de Globus et al. ${ }^{23}$. Por sua vez, segundo uma revisão integrativa de Epstein et al. ${ }^{24}$, o recurso à tecnologia digital, por meio de videochamada, é apreciada pelos pais quando não podiam permanecer na UN. Os pais do nosso estudo também valorizaram a participação ativa nos cuidados, o ambiente informal e a visita prévia à UN. Esses resultados estão em concordância com o estudo de revisão de Brett et al. ${ }^{6}$, que associou essas práticas com o aumento da confiança parental e do envolvimento com o filho.

Os resultados do presente estudo salientaram a necessidade dos profissionais reduzirem o stresse parental e otimizarem o envolvimento dos pais nos cuidados através de uma comunicação efetiva, da presença e da empatia, que são inerentes às abordagens do $\mathrm{CCF}^{25}$. Acrescentamos, ainda, que, ao confrontar os resultados nos dois GF, apesar das diferenças no tempo de internamento, não se verificaram diferenças no que concerne às práticas de educação em saúde na UN.

\section{CONCLUSÃO E IMPLICAÇÕES PARA A PRÁTICA}

Os pais consideraram como práticas de educação em saúde facilitadoras o recurso à tecnologia digital, com ênfase na disponibilização de uma app da UN pelos enfermeiros, aliada à disponibilidade para informar e explicar em um ambiente empático e informal. Esses resultados evidenciam que as tecnologias digitais não são substitutos, mas recursos complementares e promotoras das práticas de educação em saúde existentes, tendo como pilar a relação e comunicação entre os pais e os profissionais. Os enfermeiros devem incrementar os princípios das abordagens do CCF como pilar das práticas de educação em saúde dos pais na UN e recorrer a tecnologias digitais baseadas em evidência científica como recurso complementar e facilitador

Uma limitação inerente ao tipo de estudo se relaciona aos participantes incluídos poderem não ser representativos da percepção dos pais no âmbito nacional e restringir a generalização dos resultados.

\section{CONTRIBUIÇÕES DOS AUTORES}

Desenho do estudo. Nisa Rubina Pereira Souto Rosa. Maria Adriana Pereira Henriques. Maria Alice dos Santos Curado Coleta ou produção dos dados. Nisa Rubina Pereira Souto Rosa.

Análise de dados. Nisa Rubina Pereira Souto Rosa. Maria Adriana Pereira Henriques. Maria Alice dos Santos Curado Interpretação dos resultados. Nisa Rubina Pereira Souto Rosa. Maria Adriana Pereira Henriques. Maria Alice dos Santos Curado Redação e revisão crítica do manuscrito. Nisa Rubina Pereira Souto Rosa. Maria Adriana Pereira Henriques. Maria Alice dos Santos Curado

Aprovação da versão final do artigo. Nisa Rubina Pereira Souto Rosa. Maria Adriana Pereira Henriques. Maria Alice dos Santos Curado

Responsabilidade por todos os aspectos do conteúdo e a integridade do artigo publicado. Nisa Rubina Pereira Souto Rosa. Maria Adriana Pereira Henriques. Maria Alice dos Santos Curado

\section{EDITOR ASSOCIADO}

Eliane Tatsch Neves (D)

\section{EDITOR CIENTÍFICO}

Ivone Evangelista Cabral (D) 


\section{REFERÊNCIAS}

1. Gibbs DP, Boshoff K, Stanley MJ. The acquisition of parenting occupations in neonatal intensive care: a preliminary perspective. Can J Occup Ther. 2016 mar 29;83(2):91-102. http://dx.doi.org/10.1177/0008417415625421. PMid:27026720.

2. Celenza JF, Zayack D, Buus-Frank ME, Horbar JD. Family involvement in quality improvement: from bedside advocate to system advisor. Clin Perinatol. 2017 set;44(3):553-66. http://dx.doi.org/10.1016/j. clp.2017.05.008. PMid:28802339.

3. Ballantyne M, Orava T, Bernardo S, McPherson AC, Church P, Fehlings D. Parents' early healthcare transition experiences with preterm and acutely ill infants: a scoping review. Child Care Health Dev. 2017 Mar 30;43(6):783-96. http://dx.doi.org/10.1111/cch.12458. PMid:28370174.

4. Lima VF, Mazza VA. Necessidades de informações das famílias sobre saúde. Texto Contexto Enferm. 2019 Feb 14;28:e20170474. http:// dx.doi.org/10.1590/1980-265x-tce-2017-0474.

5. Smith VC. Discharge planning considerations for the neonatal intensive care unit. Arch Dis Child Fetal Neonatal Ed. 2021;106(4):442. http:// dx.doi.org/10.1136/fetalneonatal-2017-reviewers. PMid:33046524.

6. Brett J, Staniszewska S, Newburn M, Jones N, Taylor L. A systematic mapping review of effective interventions for communicating with, supporting and providing information to parents of preterm infants. BMJ Open. 2011 jun;1(1):e000023. http://dx.doi.org/10.1136/bmjopen-2010-000023. PMid:22021730.

7. Brazy JH, Anderson B, Becker P, Becker M. How parents of premature infants gather information and obtain support. Neonatal Netw. 2001;20(2):418. http://dx.doi.org/10.1891/0730-0832.20.2.41. PMid:12144111.

8. Monaghan J, Kim T, Dol J, Orovec A, Campbell-Yeo M. Parents' learning needs and preferences in a neonatal intensive care unit: a desire for enhanced communication and eHealth technology. J N Nurs. 2019 abr;26(2):101-5. http://dx.doi.org/10.1016/j.jnn.2019.09.001.

9. Skeens K, Logsdon MC, Stikes R, Ryan L, Sparks K, Hayes P et al. Health literacy and preferences for sources of child health information of mothers with infants in the Neonatal Intensive Care Unit. Adv Neonatal Care. 2016 ago;16(4):308-14. http://dx.doi.org/10.1097/ ANC.0000000000000280. PMid:27391561.

10. Mackley A, Winter M, Guillen U, Paul DA, Locke R. Health literacy among parents of newborn infants amy. Adv Neonatal Care. 2016 ago 01;16(4):283-8. http://dx.doi.org/10.1097/ANC.0000000000000295. PMid:27391562.

11. Nutbeam D, McGill B, Premkumar P. Improving health literacy in community populations: a review of progress. Health Promot Int. 2018;33(5):901-11. http://dx.doi.org/10.1093/heapro/dax015. PMid:28369557.

12. Stars I. Health literacy as a challenge for health education. SHSWeb of Conferences;2018; France. France: EDP Sciences; 2018. http://dx.doi. org/10.1051/shsconf/20184002004.

13. Moreira L. Health literacy for people-centred care: where do OECD countries stand? Paris: OECD Publishing;2018. (OECD Health Working Papers; no. 107). https://doi.org/10.1787/d8494d3a-en.
14. Lebel V, Héon M, Juneau AL, Collette K, Feeley N. The development of a digital educational program with parents of preterm infants and neonatal nurses to meet parents educational needs. J Neonatal Nurs. 2020;27(1):52-7. http://dx.doi.org/10.1016/j.jnn.2020.06.004.

15. Anwar Siani S, Dol J, Campbell-Yeo M. Impact of parent-targeted ehealth on parent and infant health outcomes. J Perinat Neonatal Nurs. 2017 out/ dez;31(4):332-40. http://dx.doi.org/10.1097/JPN.0000000000000265 PMid:28621700.

16. Orr T, Campbell-Yeo M, Benoit B, Hewitt B, Stinson J, McGrath P Smartphone and internet preferences of parents: information needs and desired involvement in infant care and pain management in the NICU. Adv Neonatal Care. 2017 abr;17(2):131-8. http://dx.doi.org/10.1097/ ANC.0000000000000349. PMid:27759567.

17. Morgan D. Basic and advanced focus groups. Washington: Sage Publications; 2018.

18. Nyumba T, Wilson K, Derrick C, Mukherjee N. The use of focus group discussion methodology: Insights from two decades of application in conservation. Methods Ecol Evol. 2018 jul;9(9):201-32. http://dx.doi. org/10.1111/2041-210X.12860.

19. Camargo BV, Justo AM. Tutorial para uso do software Iramuteq [Internet]. IRaMuTeQ; 2018 [citado 2021 mar 24]. Disponível em http://www.iramuteq.org/documentation/fichiers/Tutorial IRaMuTeQ em portugues_17.03.2016.pdf

20. Souza MAR, Wall ML, Thuler ACMC, Lowen IMV, Peres AM. The use of IRAMUTEQ software for data analysis in qualitative research. Rev Esc Enferm USP. 2018 out 04;52:e03353. http://dx.doi.org/10.1590/ S1980-220X2017015003353. PMid:30304198.

21. De Rouck S, Leys M. Information needs of parents of children admitted to a neonatal intensive care unit: a review of the literature (1990-2008) Patient Educ Couns. 2009 ago;76(2):159-73. http://dx.doi.org/10.1016/j. pec.2009.01.014. PMid:19321288.

22. Williams KG, Patel KT, Stausmire JM, Bridges C, Mathis MW, Barkin $\mathrm{JL}$. The neonatal intensive care unit: environmental stressors and supports. Int J Environ Res Public Health. 2018 jan 3;15(1):60. http:// dx.doi.org/10.3390/ijerph15010060. PMid:29301343.

23. Globus O, Leibovitch L, Maayan-Metzger A, Schushan-Eisen I, Morag I, Mazkereth $\mathrm{R}$ et al. The use of short message services (SMS) to provide medical updating to parents in the NICU. J Perinatol. 2016 maio 19;36(9):739-43. http://dx.doi.org/10.1038/jp.2016.83. PMid:27195981.

24. Epstein EG, Arechiga J, Dancy M, Simon J, Wilson D, Alhusen JL. Integrative review of technology to support communication with parents of infants in the NICU. J Obstet Gynecol Neonatal Nurs. 2017 maio/jun;46(3):357-66. http://dx.doi.org/10.1016/j.jogn.2016.11.019. PMid:28263727.

25. Gómez-Cantarino S, García-Valdivieso I, Moncunill-Martínez E, YáñezAraque B, Ugarte Gurrutxaga MI. Developing a family-centered care model in the Neonatal Intensive Care Unit (NICU): a new vision to manage healthcare. Int J Environ Res Public Health. 2020 out 1;17(19):7197. http://dx.doi.org/10.3390/ijerph17197197. 